\title{
Finite size effects in lattice RI-MOM
}

\author{
Francesco Di Renzo* \\ Università di Parma and INFN \\ E-mail: francesco.direnzo@unipr.it

\section{Michele Brambilla} \\ Università di Parma and INFN \\ E-mail: michele.brambilla@fis.unipr.it
}

RI-MOM (or its RI'-MOM variant) is one of the most polular renormalization schemes for Lattice QCD; being regulator independent, it can be effectively adopted in a lattice regularization. RI-MOM is defined in infinite volume. This is in principle a fundamental problem for the lattice, since any simulation is performed in a finite volume. From a practical point of view, one most often verifies a posteriori (by performing computations on different physical volumes) the expectation that renormalization constants, determined in the RI-MOM scheme at large momenta, should not be affected by significant finite size effects.

In the context of Numerical Stochastic Perturbation Theory, we have in recent years devised a novel method to explicitly look and correct for finite size effects (in a convenient window). We review this method, discussing how it can be applied in a non-perturbative formulation as well.

31st International Symposium on Lattice Field Theory - LATTICE 2013

July 29 - August 3, 2013

Mainz, Germany

* Speaker. 


\section{RI'-MOM: definitions and standard best practice}

Effective renormalization schemes are a key ingredient of our understanding of most quantum field theories. If renormalization is needed, there is another choice which comes first in any computation, and that is the choice of the regulator. In the continuum there is quite a consensus that dimensional regularization is an optimal choice, which in turn made $\overline{M S}$ the most used renormalization scheme for phenomenology. In the end also lattice computations have to match to the latter in order to be relevant for the high energy physics community. The regulator independent RI'-MOM renormalization scheme has established itself as an effective intermediate scheme. Being regulator independent, it can be effectively adopted both in the continuum and on the lattice.

We recall the notation in a typical case, i.e. fermionic bilinears, for which one starts by computing Green funcions

$$
G_{\Gamma}(p)=\int d x\langle p|\bar{\psi}(x) \Gamma \psi(x)| p\rangle
$$

which must then be amputated to get vertex functions

$$
\Gamma_{\Gamma}(p)=S^{-1}(p) G_{\Gamma}(p) S^{-1}(p)
$$

For the different observables, convenient projectors $\hat{P}_{O_{\Gamma}}$ have to be chosen in order to single out

$$
O_{\Gamma}(p)=\operatorname{Tr}\left(\hat{P}_{O_{\Gamma}} \Gamma_{\Gamma}(p)\right)
$$

which eventually enter the master formula defining renormalization conditions

$$
\left.Z_{O_{\Gamma}}(\mu, \alpha) Z_{q}^{-1}(\mu, \alpha) O_{\Gamma}(p)\right|_{p^{2}=\mu^{2}}=1 .
$$

The quark field renormalization constant $Z_{q}$ comes from the self-energy via

$$
Z_{q}(\mu, \alpha)=-\left.i \frac{1}{12} \frac{\operatorname{Tr}\left(\not p S^{-1}(p)\right)}{p^{2}}\right|_{p^{2}=\mu^{2}}
$$

Some twenty years ago ([1]) it was pointed out that RI'-MOM not only is a natural intermediate scheme to match the lattice to continuum, but also can be computed non-perturbatively. There is in principle nothing wrong in a lattice perturbative calculation of non-power-divergent quantities. As a matter of fact, there are a couple of quite practical limitations: lattice perturbation theory is technically hard (in particular for lattice gauge theories) and perturbative series typically converge quite badly. To overcome the latter problem, one most often makes use of a smart choice of coupling and scale [2]. One should nevertheless keep in mind that in order to fully exploit this approach at least a few orders higher than the first should be available; otherwise it amounts to only changing the value of the coupling.

In the following we will collect a few things we understood from perturbative lattice computations in the RI'-MOM scheme, in particular three-loop computations that are made possible by applying Numerical Stochastic Perturbation Theory (NSPT [3]) techniques. These are indeed in the context of perturbation theory, but we think they can offer a few interesting suggestions for 
non-perturbative computations as well.

Let us first point out three basic facts about a lattice RI'-MOM calculation.

- Renormalization conditions are imposed in the chiral limit. This means that in a nonperturbative computation a chiral extrapolation is needed.

- Every computation would be natural in momentum space. Despite that, most often the sources needed for inverting the Dirac operator are not taken diagonal in momentum. It is important to stress that, once the running dictated by Renormalization Group is taken into account, any residual momentum dependence in computation performed at different momentum scales is an irrelevant one, i.e. momentum dependence is a handle on finite lattice spacing effects.

- A conceptual key point is that RI'-MOM is in principle defined in infinite volume. One should thus worry about finite size effects.

In a typical non-perturbative computation of lattice RI'-MOM renormalization constants there are relevant counterparts to each of the previous observations. For an example of the standard best practice approach to such a computation one can inspect e.g. [4].

- Chiral extrapolations have to be carefully performed, and this is one of the major reasons for these computations being regarded as costly ones.

- Finite lattice spacing effects are often subtracted in one-loop lattice perturbation theory. Since residual effects are still there, one can fit extra (with respect to leading perturbative) irrelevant contributions.

- One must in principle consider finite size effects, but at least with the solid expectation that, being renormalization constants determined at quite large momenta, they should not be significantly affected. In the end, one has to verify that a posteriori, by repeating computations at different sizes, but this in practice a very heavy task, being the chiral extrapolation in place.

\section{NSPT for RI'-MOM renormalization constants}

It is interesting to recall how we got a first, clear hint at finite size effects in NSPT computations of renormalization constants ([5]). In Figure 1 we compare the one-loop computation of the scalar current renormalization constant for Wilson gauge action and Wilson fermions, without (left) and with (right) corrections for finite size effects. At that time one-loop finite sizes corrections were explicitely computed by evaluating the corrections to logarithmic scaling dictated by an IR cut-off.

In [6] a novel approach to the inspection of finite size effects was introduced, which has the great advantage of not asking for any modeling: there is no functional form which one needs to assume. The idea can be effectively understood ${ }^{1}$ by considering the expected form of the quark

\footnotetext{
${ }^{1}$ For a more detailed discussion of this and of most of what follows see [7].
} 

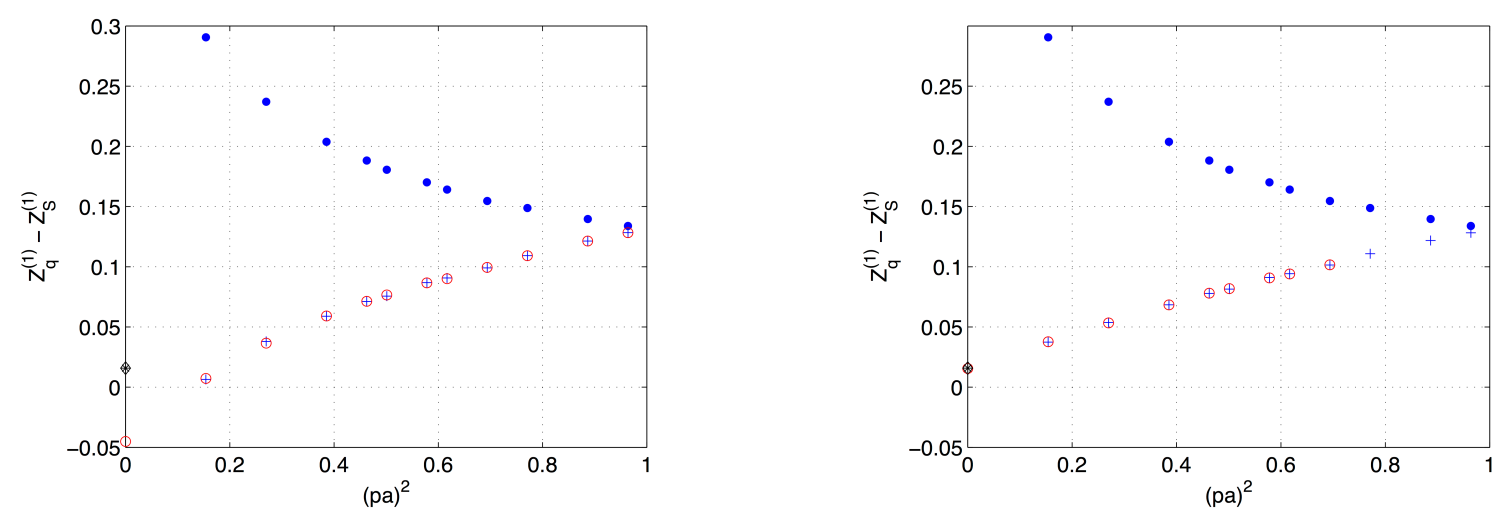

Figure 1: One-loop evaluation of the scalar current renormalization constant for Wilson gauge action and Wilson fermions, without (left) and with (right) corrections for finite size effects. Comparison to know analytical result is displayed.

self-energy on the lattice (here and in the following a hat denotes a dimensionless quantity, e.g. $\hat{p}=p a)$

$$
\hat{\Sigma}_{\gamma}=\frac{1}{4} \sum_{\mu} \gamma_{\mu} \operatorname{Tr}_{\text {spin }}\left(\gamma_{\mu} \hat{\Sigma}\right)=i \sum_{\mu} \gamma_{\mu} \hat{p}_{\mu}\left(\hat{\Sigma}_{\gamma}^{(0)}(\hat{p})+\hat{p}_{\mu}^{2} \hat{\Sigma}_{\gamma}^{(1)}(\hat{p})+\hat{p}_{\mu}^{4} \hat{\Sigma}_{\gamma}^{(2)}(\hat{p})+\ldots\right),
$$

in which the (reduced) lattice symmetries determine a tower of irrelevant contributions on top of what is expected in the continuum. In Figure 2 one can ispect the one-loop computation of the quantity ( $\bar{\mu}$ is now a given choice of a single direction)

$$
\widehat{\Sigma}_{\gamma}(\hat{p}, \bar{\mu}) \equiv-i \frac{1}{3} \frac{\operatorname{Tr}\left(\gamma_{\bar{\mu}} S^{-1}(p)\right)}{p_{\bar{\mu}}}=\hat{\Sigma}_{\gamma}^{(0)}(\hat{p})+\hat{p}_{\bar{\mu}}^{2} \hat{\Sigma}_{\gamma}^{(1)}(\hat{p})+\hat{p}_{\bar{\mu}}^{4} \hat{\Sigma}_{\gamma}^{(2)}(\hat{p})+\ldots
$$

The left panel shows the computation on a $32^{4}$ lattice. Notice that data arrange in families, how it should be clear from Eq. (2.2). Given a momentum $\left(p_{1}, p_{2}, p_{3}, p_{4}\right)$, the value of the quantities $\hat{\Sigma}_{\gamma}^{(i)}(\hat{p})$ is fixed, but one gets different values for $\widehat{\Sigma}_{\gamma}(\hat{p}, \bar{\mu})$ depending on the length of $p_{\bar{\mu}}$. The right panel displays the same quantity on both $32^{4}$ (black symbols) and $16^{4}$ (red symbols).

All in all, finite size effects are manifest because curves merge quite smoothly, but not perfectly (as it should be in infinite volume). See the lowest value of momentum on $16^{4}$ : the very same value is there also for $32^{4}$, but the two do not fall exactly on top of each other.

We can make this observation quantitative by noting that we can expect (finite size) $p L$ effects, which suggests the notation

$$
\begin{aligned}
\widehat{\Sigma}_{\gamma}(\hat{p}, p L, \bar{\mu}) & =\widehat{\Sigma}_{\gamma}(\hat{p}, \infty, \bar{\mu})+\left(\widehat{\Sigma}_{\gamma}(\hat{p}, p L, \bar{\mu})-\widehat{\Sigma}_{\gamma}(\hat{p}, \infty, \bar{\mu})\right) \\
& \equiv \widehat{\Sigma}_{\gamma}(\hat{p}, \infty, \bar{\mu})+\Delta \widehat{\Sigma}_{\gamma}(\hat{p}, p L, \bar{\mu}) \sim \widehat{\Sigma}_{\gamma}(\hat{p}, \infty, \bar{\mu})+\Delta \widehat{\Sigma}_{\gamma}(p L) .
\end{aligned}
$$

Due to the approximation which is entailed in the last line (we neglect finite $a$ corrections on top of finite $L$ corrections) and since $p_{\mu} L=\frac{2 \pi n_{\mu}}{L} L=2 \pi n_{\mu}$, one easily realizes that there is only one 

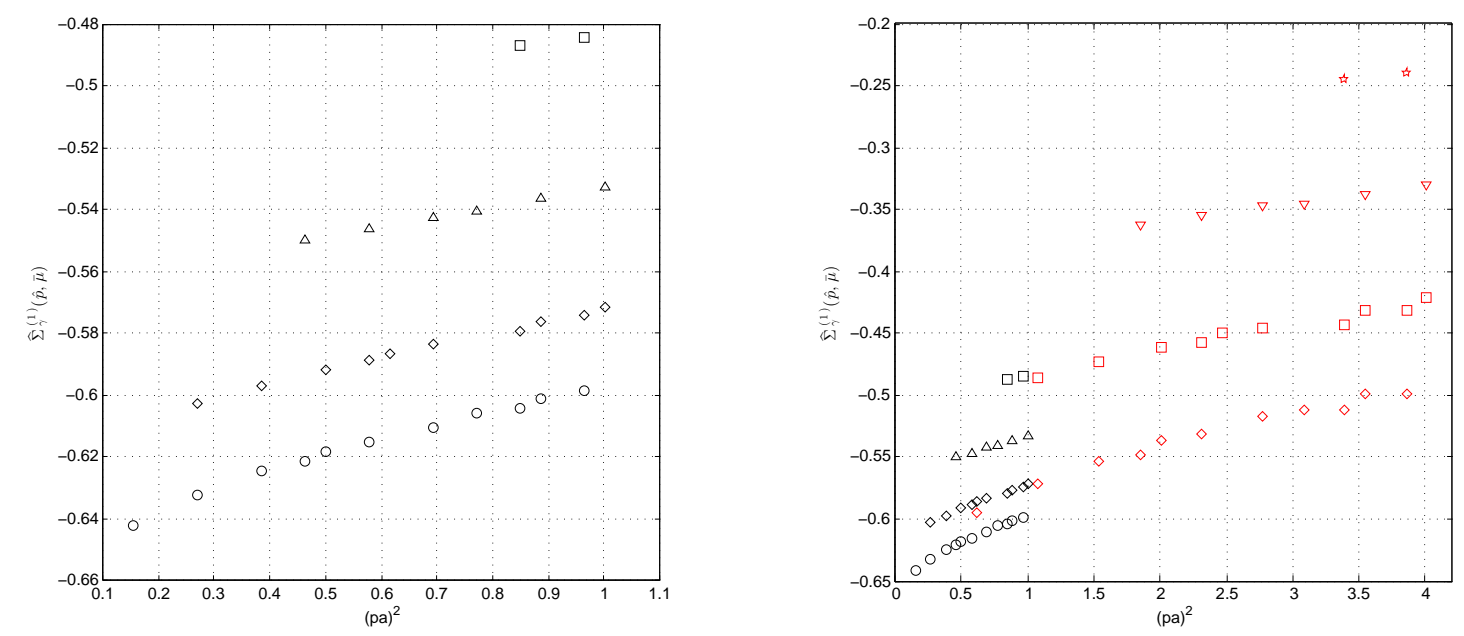

Figure 2: One-loop evaluation of $\widehat{\Sigma}_{\gamma}(\hat{p}, \bar{\mu})$ (see Eq. (2.2)) on a $32^{4}$ lattice (left) and on both a $32^{4}$ and a $16^{4}$ lattice (right).

finite size correction for each 4-tuple $n_{\mu}$ and no functional form has to be inferred for the correction.

In [7] we show all this at work for the computation of quark currents renormalization constants for the lattice regularization defined by Tree Level Symanzik improved gauge action and two flavors of Wilson fermions. The quantities

$$
\left.\widehat{O}_{\Gamma}(\hat{p}, p L, \bar{\mu}) \equiv \frac{\widehat{\Sigma}_{\gamma}(\hat{p}, p L, \bar{\mu})}{\hat{O}_{\Gamma}(\hat{p}, p L)}\right|_{\log \text { subrr }}
$$

can be computed, whose extrapolation to zero momentum provides our determination of renormalization constants as defined in Eq. (1.3): the numerator takes the quark renormalization into account, while the subtrction of the logarithmic corrections is possible via a determination of the latter from continuum computations ${ }^{2}$. In the denominator one can recognize the lattice counterparts of Eq. (1.1).

The quantities $\widehat{O}_{\Gamma}(\hat{p}, p L, \bar{\mu})$ can be computed on different lattice sizes and fitted to formulas in which momentum dependence is taken into account in the form of expansions in hypercubic invariants and one finite size correction is there for every 4-tuple (i.e. a single correction is there for all the momenta which are obtained with a single choice of the 4-tuple on different sizes).

Figure 3 shows how effective the correction for finite size effects can be. In the left panel, the one-loop $\widehat{O}_{\Gamma}(\hat{p}, p L, \bar{\mu})$ for the scalar current is plotted as measured on $32^{4}$ and $16^{4}$ lattices, with no corrrection for finite size effects. In the right panel, the latter are subtracted, after having being fitted as explained above. The interested reader can inspect in [7] that one-loop results are obtained which agree with analytical ones and that we can push our computations to three-loop (we can not go beyond three-loop because this is the order at which anomalous dimensions are known; as said,

\footnotetext{
${ }^{2}$ For details see [7].
} 

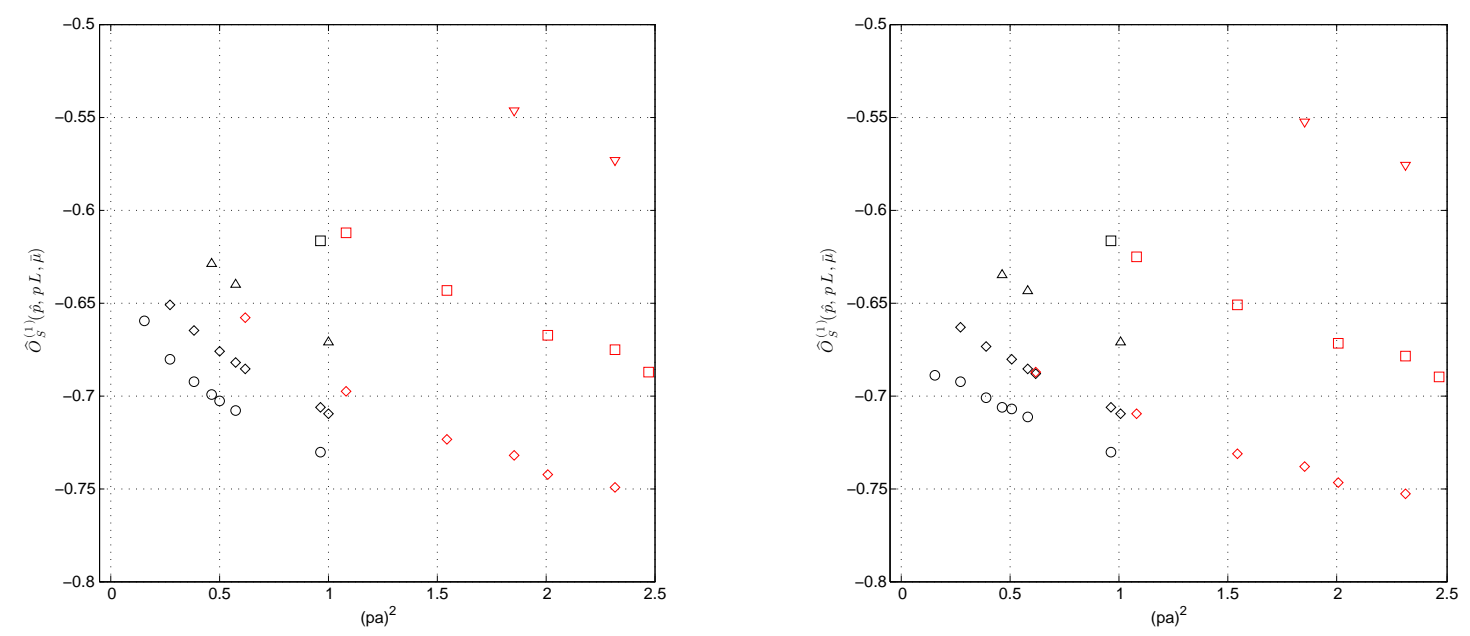

Figure 3: One-loop $\widehat{O}_{S}(\hat{p}, p L, \bar{\mu})$ (see Eq. (2.4)) measured on a $32^{4}$ (black) and a $16^{4}$ (red) lattice, without (left) and with (right) finite size corrections.

the latter are obtained from the continuum computations).

All in all, Figure 3 displays finite size corrections that are clearly visible in the left panel: families corresponding to the same length of $p_{\bar{\mu}}$ (these are plotted using the same symbols, i.e. circles, diamonds, triangles and so on) do not fall smoothly on top of each other. After we correct for finite size effects (right panel), they do.

\section{A better best practice?}

There is in principle no reason why one should not try the same approach to finite size effects in a non-pertubative computation. We are not claiming that finite size effects should be so big in non-perturbative computations as they showed up in our perturbative framework: IR effects are simply not supposed to be the same. Still, it is the methodology which could be given a try. What we would say is that there could be more finite size effects information in RI'-MOM computations than it is usually singled out, and that could be got with our method. We do plan to give this approach a try.

All in all, a possible approach to merge NSPT and non-perturbative (NP) computations of renormalization constants could entail the following:

- A NSPT high-order computation;

- a check and (possibly) correction for finite size effects for both NSPT and NP results;

- in this way, a better subtraction of irrelevant effects could be implemented.

Something like this can be recognized in [8]. 
Notice that it could in any case turn out that there are cases in which a high-order NSPT computation is not less precise than a NP determination.

\section{Acknowledgments}

A lot of people made our understanding better and better in the long process that eventually resulted in [7] and in a similar computation for Iwasaki gauge action and four flavors of Wilson fermions that is going to be published soon (in collaboration with M. Hasegawa). Among them, M. Bonini, V. Lubicz, C. Tarantino, R. Frezzotti, P. Dimopoulos and H. Panagopoulos.

This research is supported by the Research Executive Agency (REA) of the European Union under Grant Agreement No. PITN-GA-2009-238353 (ITN STRONGnet). We acknowledge partial support from both Italian MURST under contract PRIN2009 (20093BMNPR 004) and from I.N.F.N. under i.s. MI11 (now QCDLAT).

\section{References}

[1] G. Martinelli, C. Pittori, C.T. Sachrajda, M. Testa and A. Vladikas, A general method for non perturbative renormalization of lattice operators, Nucl. Phys. B 445 (1995) 81 [hep-lat/9411010].

[2] G. P. Lepage and P. Mackenzie, On the viability of lattice perturbation theory, Phys. Rev. D48 (1993) 2250 .

[3] F. Di Renzo, E. Onofri, G. Marchesini and P. Marenzoni, Four loop result in SU(3) lattice gauge theory by a stochastic method: Lattice correction to the condensate, Nucl. Phys. B 426, 675 (1994). F. Di Renzo and L. Scorzato, Numerical Stochastic Perturbation Theory for full QCD, JHEP 04 (2004) 073.

[4] M. Constantinou et al. [ETM Collaboration], Non-perturbative renormalization of quark bilinear operators with $N_{f}=2$ (tmQCD) Wilson fermions and the tree-level improved gauge action, JHEP 1008, 068 (2010).

[5] F. Di Renzo, V. Miccio, L. Scorzato and C. Torrero, High-loop perturbative renormalization constants for Lattice QCD. I. Finite constants for Wilson quark currents, Eur. Phys. J. C 51, 645 (2007).

[6] F. Di Renzo, E. -M. Ilgenfritz, H. Perlt, A. Schiller and C. Torrero, Two-point functions of quenched lattice QCD in Numerical Stochastic Perturbation Theory. (I) The ghost propagator in Landau gauge, Nucl. Phys. B 831, 262 (2010).

F. Di Renzo, E. -M. Ilgenfritz, H. Perlt, A. Schiller and C. Torrero, Two-point functions of quenched lattice QCD in Numerical Stochastic Perturbation Theory. (II) The Gluon propagator in Landau gauge, Nucl. Phys. B 842, 122 (2011).

[7] M. Brambilla and F. Di Renzo High-loop perturbative renormalization constants for Lattice QCD (II): three-loop quark currents for tree-level Symanzik improved gauge action and $n_{f}=2$ Wilson fermions, arXiv:1310.4981.

[8] J. Simeth, A. Sternbeck, E. -M. Ilgenfritz, H. Perlt and A. Schiller, Discretization Errors for the Gluon and Ghost Propagators in Landau Gauge using NSPT, these proceedings (arXiv:1311.1934). 